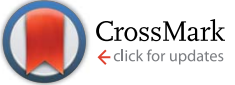

Cite this: RSC Adv., 2017, 7, 17184

\title{
Photochirogenic nanosponges: phase-controlled enantiodifferentiating photoisomerization of (Z)-cyclooctene sensitized by pyromellitate- crosslinked linear maltodextrin $\uparrow$
}

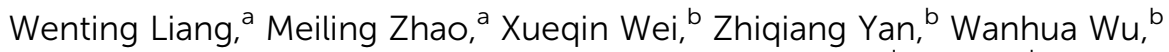 \\ Fabrizio Caldera, ${ }^{c}$ Francesco Trotta, ${ }^{\star c}$ Yoshihisa Inoue, ${ }^{\star d}$ Dan Su, ${ }^{\text {b }}$ Zhihui Zhong ${ }^{\mathrm{b}}$ \\ and Cheng Yang*b
}

\begin{abstract}
Linear maltodextrin (LM) was cross-linked by pyromellitic dianhydride to afford LM polymers of different cross-linking degrees. When soaked in water, these cross-linked LM polymers (nanosponges (NSs)), evolved into several phases from sol to suspension, then to flowing gel, and finally to rigid gel with an increase in their content. Enantiodifferentiating photoisomerization of (Z)-cyclooctene (1Z) to chiral (E)-isomer (1E), which was employed as a benchmark reaction to quantitatively assess the environmental-to-molecular chirality transfer process, was performed in aqueous media containing these pyromellitate-crosslinked LM-NSs in different phases. The enantiomeric excess (ee) of $1 \mathrm{E}$ obtained was relatively insensitive to the phases at least up to the flowing gel phase, but became highly sensitive in the rigid gel phase, exhibiting an abrupt drop in the early rigid gel phase followed by a rapid recovery in the late rigid gel phase. A comparison with the phase-dependent ee profiles previously reported for similar pyromellitate-crosslinked cyclodextrin (CD)- and cyclic nigerosylnigerose (CNN)-NSs revealed that the chiral void space created around the pyromellitate linker in NS is responsible for the dramatic changes in ee in the rigid gel phase, whereas the inherent host cavity in CD/CNN plays only limited roles in the supramolecular photochirogenesis mediated by the sensitizer-crosslinked NSs. The latter insight allows us to further expand the applicable range of the present concept and methodology by employing a much wider variety of oligosaccharides as well as substrates and sensitizing cross-linkers.
\end{abstract}

\author{
Received 14th December 2016 \\ Accepted 24th February 2017 \\ DOI: 10.1039/c6ra28211c \\ rsc.li/rsc-advances
}

\section{Introduction}

Chiral photochemistry (photochirogenesis), which is employed to induce molecular chirality in an excited-state reaction, is an attractive yet challenging area of chemistry, which provides environmentally benign direct access to optically active molecules with unconventional, often constrained, skeletons that are difficult or tedious to construct via thermal asymmetric synthesis. $^{1-6}$ However, controlling the stereochemical

aInstitute of Environmental Sciences, Department of Chemistry, Shanxi University, Taiyuan 030006, China

${ }^{b}$ State Key Laboratory of Biotherapy, West China Medical School, Key Laboratory of Green Chemistry, Technology of Ministry of Education, College of Chemistry, Sichuan University, 29 Wangjiang Road, Chengdu 610064, China. E-mail: yangchengyc@scu.edu.cn

'Department of Chemistry, University of Torino, Via P. Giuria 7, 10125 Torino, Italy. E-mail: francesco.trotta@unito.it

${ }^{d}$ Department of Applied Chemistry, Osaka University, 2-1 Yamada-oka, Suita 5650871, Japan. E-mail: inoue@chem.eng.osaka-u.ac.jp

$\dagger$ Electronic supplementary information (ESI) available: Thermogravimetric analyses, dynamic light scattering data for LM-NS 7, optical microscope images of LM-NS 7, and SEM images of LM-NSs 7 and 8. See DOI: 10.1039/c6ra28211c consequence of a chiral photoreaction is not a simple task, in particular, for enantiodifferentiating photosensitization reaction, where only short-lived weak intermolecular stereochemical interactions are available and the electronically excited states generated are highly reactive. ${ }^{7}$ Indeed, the enantioselectivities reported for many of the enantiodifferentiating photosensitization reactions are modest, ${ }^{1-6}$ which are not inherent and are due to our lack of comprehensive understanding of the factors and mechanism that control the enantiodifferentiating photosensitization process.

The supramolecular approach to photochirogenesis provides a convenient promising tool for facilitating the excited-state chirality transfer from an enantiomeric host to prochiral substrate through the long-lasting intimate supramolecular contacts of guest substrate(s) with a chiral host in the ground as well as excited state. ${ }^{2-15}$ A variety of chiral supramolecular hosts including cyclodextrins, ${ }^{16-23}$ chirally modified zeolite supercages, ${ }^{24-26}$ biomolecules, ${ }^{27,28}$ chiral hydrogen-bonding templates, ${ }^{29-31}$ and rotaxanes ${ }^{32}$ have hitherto been examined to achieve modest to high enantioselectivities. More recently, chiral supramolecular assemblies such as gels ${ }^{\mathbf{3 3 , 3 4}}$ and liquid 


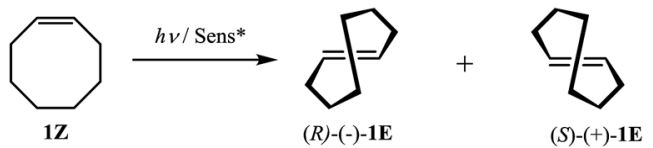

Scheme 1 Enantiodifferentiating photoisomerization of $1 Z$ to chiral $1 \mathrm{E}$.

crystals $^{35}$ have also been employed as chiral environments for supramolecular photochirogenesis.

We recently reported the results of a series of studies on the phase-dependent enantiodifferentiating photoisomerization of $(Z)$-cyclooctene (1Z) (Scheme 1) mediated by pyromellitatecrosslinked polymers of cyclic oligosaccharides with different cavity diameters and depths, i.e. $\beta$ - and $\gamma$-cyclodextrins $(\mathrm{CDs})^{36}$ and cyclic nigerosylnigerose (CNN), ${ }^{37,38}$ as shown in scheme 2 . These cross-linked polymers showed intriguing swelling behaviour upon soaking in water and hence were named nanosponges (NSs). ${ }^{39-41}$ Both the CD-based 2-4 and CNN-based NSs 5 and 6 (Scheme 2) evolved into several phases from sol to

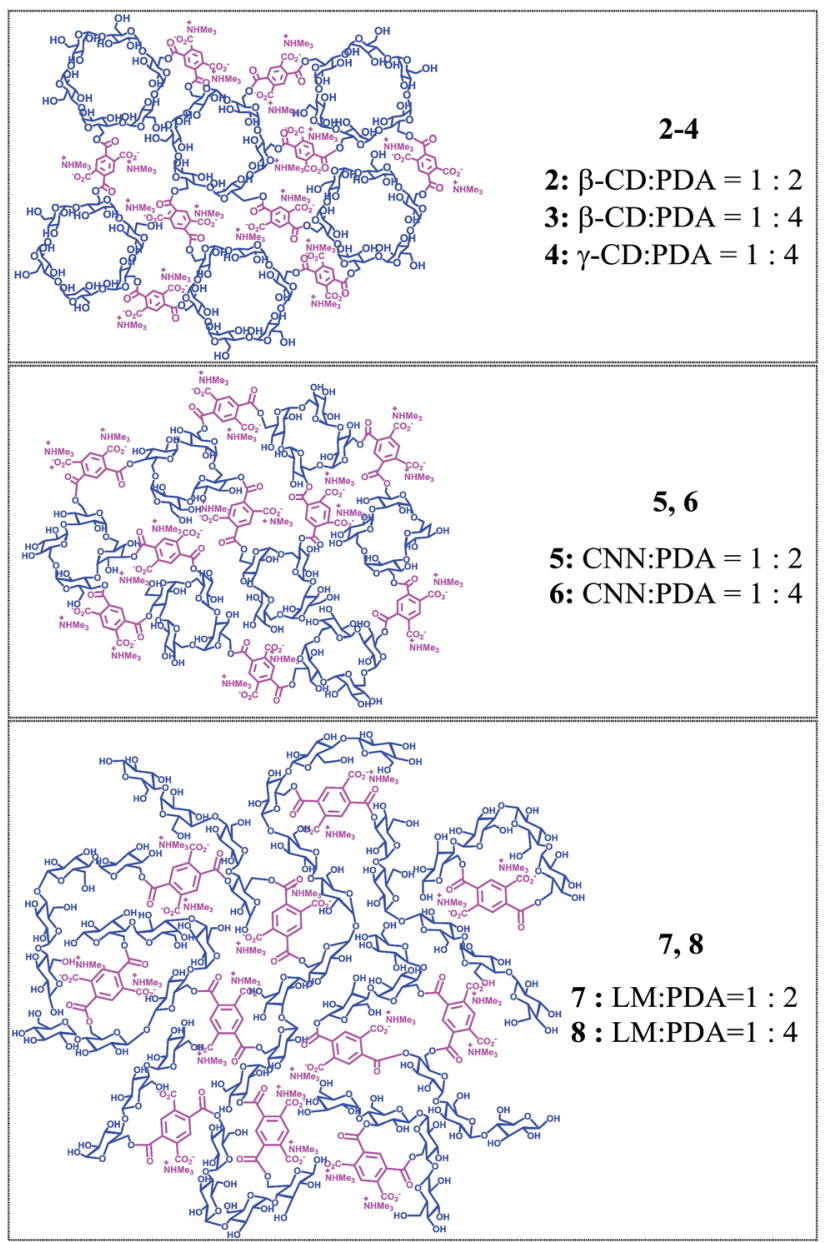

Scheme 2 Chemical structures of CD-NSs 2-4, CN-NNSs 5, 6, and LM-NS 7, 8 synthesized using different oligosaccharide : linker ratios. CD: cyclodextrin; CNN: cyclic nigerosylnigerose; LM: linear maltodextrin; and PDA: pyromellitic dianhydride. rigid gel via suspension and flowing gel upon a gradual increase in the NS content in water and more crucially served as effective chiral sensitizers for the enantiodifferentiating photoisomerization of $\mathbf{1 Z}$ to chiral $(E)$-isomer (1Z).$^{\mathbf{3 6}, 37}$ Interestingly, the enantiomeric excess (ee) of 1E obtained upon the photosensitization of $\mathbf{1 Z}$ with NS was critically affected by the phase condition. This phase-dependent photochirogenic behaviour had never been observed for the non-polymeric cyclic oligosaccharides-based sensitizers reported earlier. ${ }^{\mathbf{4 2 - 4 5}} \mathrm{We}$ proposed a progressive aggregation mechanism to rationalize this phase-dependent behaviour of the ee product. For CDbased NS, the interparticle complexation between the pyromellitate linker and the CD host exposed on the surfaces of neighbouring CD-NS particles is deemed to drive the progressive aggregations, leading to phase transitions upon the gradual increase in the CD-NS concentration. As a cyclic tetrasaccharide, CNN is much smaller in ring size than CDs and bears only a shallow concave. Apparently, this concave does not effectively function as a binding site and instead intra- and/or interparticle voids were thought to function as the binding and sensitizing sites to influence the stereochemical outcomes. The size, shape, and flexibility of these polymer voids are highly sensitive to the degree of aggregation or the phase of the medium and hence are considered to be responsible for the phase-dependent ee values. These considerations led us to two further questions concerning the general validity of the phenomenon and the roles of the molecular host (CD and CNN) incorporated in NSs: (1) how widely the phase-control concept is applicable in supramolecular photochirogenesis and (2) to what extent the molecular host used as a building block contributes to the photochirogenesis in the cross-linked oligosaccharide polymer.

In the present study, to address these issues and also to further expand our understanding of the relationship between the phase evolution and the stereochemical outcome of the photosensitized enantiodifferentiation, we employed linear maltodextrin (LM) as a catenulate oligosaccharide building block, which has no inherent cavity or concave and hence is inclusion-free, and pyromellitic dianhydride (PDA) as a crosslinking agent to obtain a pyromellitate-crosslinked linear maltodextrin polymer (LM-NS). The photochirogenic performance of LM-NSs 7 and 8 upon enantiodifferentiating photosensitization of $\mathbf{1 Z}$ was examined in different phases evolved in water, and the results have been comparatively discussed with those obtained using similar pyromellitate-crosslinked CD- and CNNNSs (2-6) (Scheme 2).

\section{Results and discussion}

\section{Syntheses}

Two LM-NS sensitizers with different degrees of cross-linking ( 7 and 8) were prepared by reacting LM with PDA in the presence of trimethylamine in DMSO at room temperature and purified by extensive Soxhlet extraction of the resulting polymer with acetone (Scheme 2); note that the formal molar ratios indicated herein were calculated as the ratios of seven glucose units (equivalent to $\beta$-CD) to PDA. The LM-NSs thus obtained were 
characterized by thermal analyses, FT-IR, elemental analyses, and swelling properties. In this reaction, two carboxylic acid moieties remain on the pyromellitate linker, a significant fraction of which forms a salt with trimethylammonium ion and is partially ionized in aqueous media.

\section{Chiroptical properties}

$\beta$-CD possesses a hydrophobic cavity that can include one $\mathbf{1 Z}$ molecule with a relatively high binding affinity. ${ }^{40,41}$ In contrast, LM has no defined binding site for accommodating 1Z. However, the combined use of the conformationally flexible hydrophilic LM and rigid hydrophobic linker is expected to leave some void space, particularly around the pyromellitate moiety, in the resulting loosely packed cross-linking LM polymer. In this LM-NS, the pyromellitate moiety plays triple roles of a cross-linker, pillar, and photosensitizer, whereas the inherently chiral LM segments nearby provide the chiral environment for effecting the enantiodifferentiating photoisomerization of prochiral $\mathbf{1 Z}$ to chiral $\mathbf{1 E}$ sensitized by pyromellitate.

To evaluate the chiral environment induced around the pyromellitate linker, UV-vis and circular dichroism spectral examinations of LM-NSs (7 and 8) were performed in aqueous solutions. As shown in Fig. 1b and d, both 7 and 8 exhibit negative Cotton effect for the ${ }^{1} \mathrm{~L}_{\mathrm{a}}$ band of pyromellitate (at 265 and $268 \mathrm{~nm}$, respectively) in pure water. The anisotropy factors ( $g=\Delta \varepsilon / \varepsilon$, where $\Delta \varepsilon$ denotes the molar circular dichroism and $\varepsilon$ denotes the molar extinction coefficient) observed for LM-NSs are smaller than those reported for CD-NSs, ${ }^{36}$ whereas slightly larger than those reported for $\mathrm{CNN}^{-N_{S s}{ }^{37}}$ under comparable measuring conditions. These induced circular dichroism (ICD) signals clearly indicate that the pyromellitate chromophore is located in a chiral environment and hence is applicable to chirality transfer through photosensitization.

\section{Chiral environment}

To assess the conformational flexibility of the cross-linked polymers, the effects of methanol added to the aqueous

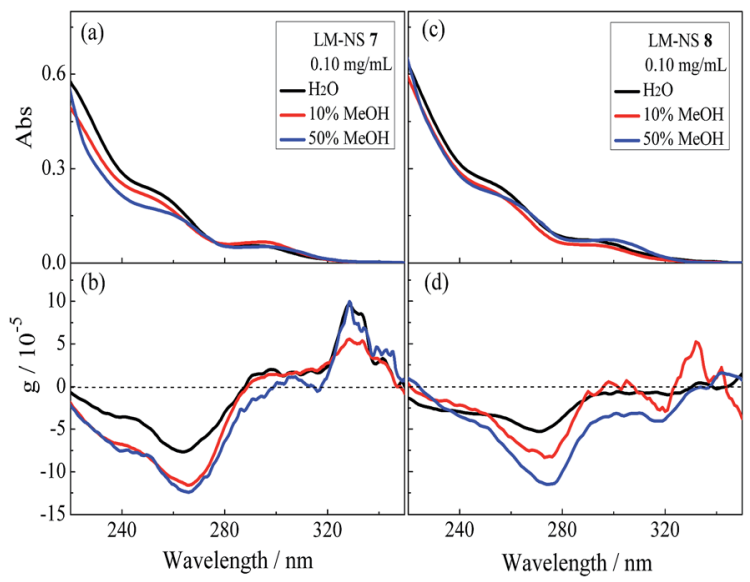

Fig. 1 UV-vis (a and c) and anisotropy $(g=\Delta \varepsilon / \varepsilon$ ) factor (b and $\mathrm{d}$ ) spectra of LM-NS $7\left(0.10 \mathrm{mg} \mathrm{mL}^{-1}\right)$ and LM-NS $8\left(0.10 \mathrm{mg} \mathrm{mL}^{-1}\right)$ in aqueous solutions containing $0 \%$ (black), 10\% (red), and $50 \%$ (blue) methanol at ambient temperature. solutions of 7 and 8 on their chiroptical properties were examined via UV-vis and circular dichroism spectroscopies. As shown in Fig. 1, the addition of $10-50 \%$ methanol led to appreciable decreases in absorbance in UV-vis and increases in anisotropy in the circular dichroism spectra, but the original peak position and shape of the main band were maintained, thus implying that the cross-linked polymers have a relatively rigid framework and the addition of an organic solvent does not significantly alter the orientation and conformation of the pyromellitate moieties. Subsequent addition of $\mathbf{1 Z}(3 \mathrm{mM})$ to the isotropic LMNS solutions did not cause any appreciable changes in the circular dichroism spectra, for which, the lack of defined hydrophobic binding sites and relatively rigid framework of the cross-linked LM-NS would be responsible. In summary, the microenvironmental chirality created around the pyromellitate sensitizer in LM-NS is manipulable to certain extent through the orientational and/or conformational changes caused upon the addition of methanol, but is not altered by substrate $\mathbf{1 Z}$.

\section{Phase evolution}

We demonstrated that both CD-NSs 2-4 (ref. 36) and CNN-NSs 5, 6 (ref. 37) evolve into a series of phases changing from sol to rigid gel upon a gradual increase in the polymer content in water. Interestingly, the enantiomeric excess (ee) of $\mathbf{1 E}$ produced upon photosensitization by 2-6 critically varies with the polymer content, i.e. the phase evolved, exhibiting the most remarkable changes around the border of flowing gel to rigid gel. This result led us to the idea that the NS void space for photochirogenesis is manipulable via phase evolution.

To assess the aggregation and phase-evolution behaviour of LM-NS in water at room temperature, the contents of 7 and $\mathbf{8}$ were gradually increased and the appearance of the resulting solutions was observed. As shown in Fig. 2a, both 7 and 8 exhibit a stepwise phase-evolution from transparent sol to suspension, then to flowing gel, and finally to rigid gel, as was

a)

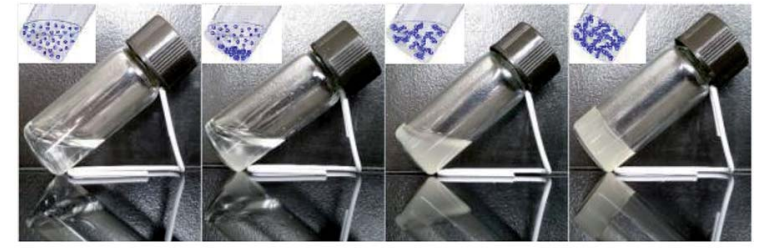

b)

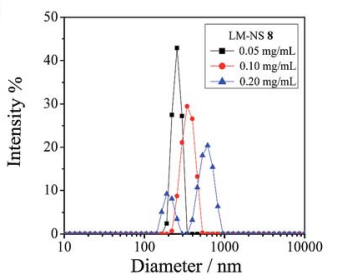

c)

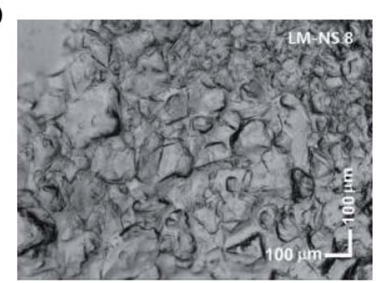

Fig. 2 (a) Images of aqueous solutions of LM-NS 8 at 0.1 (sol), 10 (suspension), 125 (flowing gel), and $245 \mathrm{mg} \mathrm{mL}^{-1}$ (rigid gel) (from left to right). Inset: schematic of the phase evolution of 8 . (b) Dynamic light scattering (DLS) intensities measured at 0.05 (black), 0.1 (red), and 0.2 (blue) $\mathrm{mg} \mathrm{mL}^{-1}$ concentrations of LM-NS 8. (c) Optical microscopy image of LM-NS 8 at $245 \mathrm{mg} \mathrm{mL}^{-1}$. 
Table 1 Enantiodifferentiating photoisomerization of $1 \mathrm{Z}$ to chiral $1 \mathrm{E}$ sensitized by LM-NSs 7 and 8 in various phases developed in aqueous media containing $0-50 \%$ methanol $^{a}$

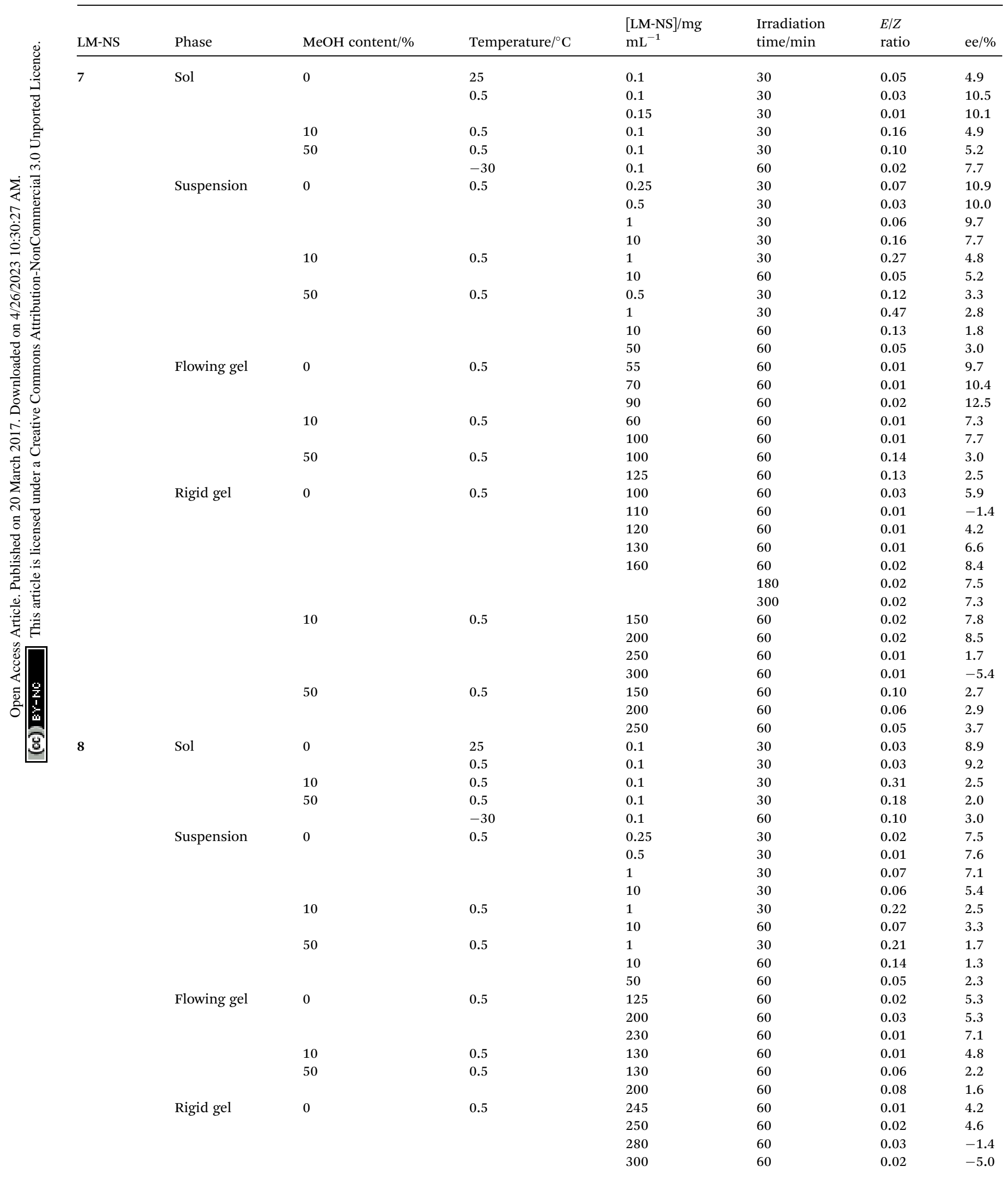


Table 1 (Contd.)

\begin{tabular}{|c|c|c|c|c|c|c|c|}
\hline LM-NS & Phase & $\mathrm{MeOH}$ content $/ \%$ & Temperature $/{ }^{\circ} \mathrm{C}$ & $\begin{array}{l}{[\mathrm{LM}-\mathrm{NS}] / \mathrm{mg}} \\
\mathrm{mL}^{-1}\end{array}$ & $\begin{array}{l}\text { Irradiation } \\
\text { time/min }\end{array}$ & $\begin{array}{l}E / Z \\
\text { ratio }\end{array}$ & ee $/ \%$ \\
\hline & & & & 350 & 60 & 0.02 & -11.1 \\
\hline & & & & 500 & 60 & 0.01 & 5.8 \\
\hline & & & & 575 & 60 & 0.01 & 5.9 \\
\hline & & 10 & 0.5 & 250 & 60 & 0.02 & 4.2 \\
\hline & & & & 500 & 60 & 0.01 & 4.7 \\
\hline & & 50 & 0.5 & 250 & 60 & 0.07 & 1.5 \\
\hline & & & & 300 & 60 & 0.06 & 2.2 \\
\hline & & & & 500 & 60 & 0.04 & 2.4 \\
\hline
\end{tabular}

exactly the case with the previously reported CD- and CNN-NSs 2-6. For the less cross-linked LM-NS 7, the critical concentrations for sol-suspension, suspension-flowing gel, and flowing gel-rigid gel phase-transitions were $0.15,55$, and $100 \mathrm{mg} \mathrm{mL}^{-1}$, respectively. These critical phase-transition concentrations were lower than the corresponding values $(0.25,125$, and $245 \mathrm{mg} \mathrm{mL}{ }^{-1}$, respectively) determined for the more crosslinked LM-NS 8. This is presumably due to the smaller amount of LM segments exposed on the surface of $\mathbf{8}$, which causes the aggregation of the cross-linked polymers via hydrogen-bonding interactions.

Apart from the critical phase-transition concentrations, the overall phase-evolution behaviours of 7 and 8 resemble each other. Typically, LM-NS 8 smoothly dissolved in water to afford an isotropic solution up to $0.25 \mathrm{mg} \mathrm{mL}^{-1}$ concentration, after which, transparent precipitates started to appear (Fig. 2a). Even in the apparently homogeneous solution, the polymer started to aggregate at relatively low concentrations; thus, the mean hydrodynamic diameter evaluated by dynamic light scattering (DLS) gradually increased from 250 to 350 and then to $600 \mathrm{~nm}$ with an increase in the concentration from 0.05 to 0.1 and then to $0.2 \mathrm{mg} \mathrm{mL}^{-1}$ (Fig. 2b). The solution obviously became turbid at $0.25 \mathrm{mg} \mathrm{mL}^{-1}$ to afford a suspended solution. When the concentration was further increased to $125 \mathrm{mg}$ $\mathrm{mL}^{-1}$, the turbid solution started to gelate to provide a gel-like binary phase composed of liquid and gel, or the 'flowing gel' state, which was eventually converted to rigid gel at a critical gelation concentration of $245 \mathrm{mg} \mathrm{mL} \mathrm{m}^{-1}$ (Fig. 2c). Optical microscopic (Fig. 2c and S6 $\dagger$ ) and SEM (Fig. S7 and S8 $\dagger$ ) investigations of the rigid gel phase revealed the adhesive nature of the gel with the particle sizes in the range of 10$100 \mathrm{~nm}$. These results indicate that the phase evolution is a continuous growth process, in which the size of the aggregates gradually increases to form precipitates, which further adhere to afford gels (as illustrated in the inset of Fig. 2a). The abundant hydroxyl groups of the LM moieties in LM-NS are likely to dominate the progressive interparticle adhesion via the hydrogen-bonding interaction.
The concentration-dependent stepwise phase-transitions of LM-NS also occurred in aqueous solutions containing $10-50 \%$ methanol. The phase transition concentrations in aqueous methanol were higher than the corresponding values observed in pure water. LM-NS 7 exhibited the phase transition concentrations for suspension, flowing gel and rigid gel at $0.25,60$ and $150 \mathrm{mg} \mathrm{mL}^{-1}$, respectively, in 1:9 and 1:1 methanol-water. More crosslinked LM-NS 8 also showed comparable transition concentrations at $0.5,130$ and $250 \mathrm{mg} \mathrm{mL}^{-1}$, respectively, in $1: 9$ and $1: 1$ methanol-water.

\section{Photochirogenesis in LM-NS}

Enantiodifferentiating photoisomerization of $\mathbf{~ Z ~ s e n s i t i z e d ~ b y ~} 7$ and 8 was carried out under different phase conditions in aqueous media containing $0 \%, 10 \%$, and $50 \%$ methanol. Since the photosensitizations with 7 and $\mathbf{8}$ gave higher or comparable ee in solution phase at $0.5^{\circ} \mathrm{C}$ than at $25^{\circ} \mathrm{C}$ (Table 1), practically, all the experiments were performed at $0.5^{\circ} \mathrm{C}$.

In isotropic pure water solution, both $\mathbf{7}$ and $\mathbf{8}$ afforded $(S)-\mathbf{1 E}$ in comparable $10.5 \%$ and $9.2 \%$ ee. In $10-50 \%$ methanolic water, the ee of $1 \mathrm{E}$ decreased to $4.9-5.2 \%$ ee for 7 and to $2.0-$ $2.5 \%$ ee for 8 , which were however appreciably improved to $7.7 \%$ and $3.0 \%$ by lowering the temperature to $-30{ }^{\circ} \mathrm{C}$ (Table 1 , top lines). These results indicate that despite the fact that the same building monomer and linker were employed, the different cross-linking degrees and framework rigidity afforded diverse chiral environments for the enantiodifferentiating photoisomerization of $\mathbf{1 Z}$, which is readily tunable by adjusting the external factors such as solvent composition and temperature.

Intriguingly, the enantioselectivity was a critical function of the phase condition. As shown in Fig. 3f, the ee value of $\mathbf{1 E}$ obtained upon sensitization with 7 in pure water was practically kept constant at $10 \pm 2 \%$ with some fluctuations through the solution to the late flowing gel phase, but suddenly reduced and sign-inverted to reach a minimum $(-1.4 \%)$ in the early rigid gel phase, and finally recovered to $+8.4 \%$ (or $7.3-7.5 \%$ upon prolonged irradiations) in the late rigid gel phase, presumably due 
to the structural evolution of rigid gel. Moreover, upon sensitization with 8 (Fig. $3 \mathrm{~g}$ ), the ee behaved very similarly, remaining at $7.0 \pm 1.5 \%$ up to the late flowing gel phase, suddenly reduced and sign-inverted to attain $-\mathbf{1 1 . 1 \%}$ in the early rigid gel phase, and then recovered to $+5.9 \%$ in the late rigid gel phase.

These ee profiles resemble those observed for CD-NSs (except $\gamma$-CD-based NS 4) and CNN-NSs (Fig. 3a-e), which also show a sudden drop (sometimes with accompanying inversion of product chirality) near the flowing-rigid gel border. This is rather unexpected because in contrast to the CD- and CNNanalogues, LM-NS does not bear any inherent cavity or concave in the cross-linked polymer structure. Previously, we proposed that the sudden change in ee observed at the phase border in the photosensitization of $\mathbf{1 Z}$ with CD- and CNN-NSs 26 is driven by the switching of the photosensitizing site from the cavity or concave of the cyclic oligosaccharide CD or CNN to the chiral void space surrounded by the outside walls of CD or CNN.

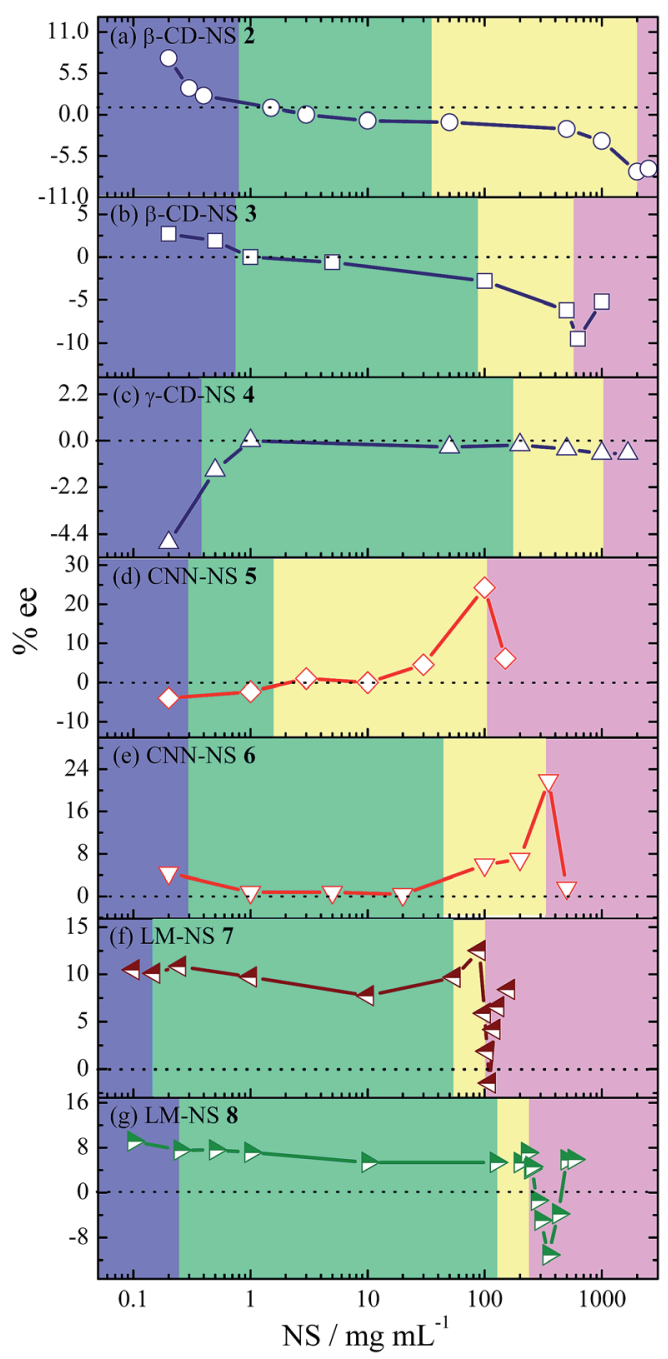

Fig. 3 ee profiles of $1 \mathrm{E}$ obtained upon enantiodifferentiating photoisomerization of $1 Z$ sensitized by (a) $\beta$-CD-NSs $2,{ }^{36}$ (b) $3,{ }^{36}$ (c) $\gamma$-CD-NS $4,^{36}$ (d) CNN-NSs $5,{ }^{37}$ (e) $6,{ }^{37}$ (f) LM-NSs 7 and (g) 8 at various concentrations and in various phases. The violet, green, yellow, and pink regions represent the sol, suspension, flowing gel, and rigid gel phases, respectively.
Obviously, no defined binding site exists in the catenulate oligosaccharide LM-based NSs 7 and 8, revealing that our previously proposed switching mechanism is not valid and further implying that the phase-dependent chirality is a more universal phenomenon for photosensitization in hydrogel, regardless of the building block employed.

To better understand the phase-dependent photochirogenesis behaviour, we examined the chiroptical properties of LM-NS in two extreme phases. As can be seen from Fig. 4, the circular dichroism spectra of LM-NS 7 in all the phases (from solution to rigid gel) exhibit simple negative Cotton effects for the main band of the pyromellitate linker, the anisotropy $(g)$ factors of which are $\sim 10^{-4}$ at the extremum (260-270 nm), a typical value for the allowed $\pi-\pi^{*}$ transition. The lack of exciton couplets at the main band implies that the pyromellitate chromophores are spatially separated by the LM moieties in the cross-linked polymer LM-NS. However, the $g$ factor at $271 \mathrm{~nm}$ gradually decreases in the sol and suspension phases and more rapidly in the gel phases (Fig. 4 inset). This means that the chiral environment created around the pyromellitate linker by the surrounding LM moieties differs in each phase. Since the enantiodifferentiating photoisomerization of $\mathbf{1 Z}$ occurs upon sensitization by pyromellitate, the ee of $\mathbf{1 E}$ thus produced should be sensitive to the chiral environment created in the photosensitization site, which is likely to be a polymer void space encircled by a pyromellitate and several LM moieties. This mechanism based on the chiroptical property change of the LMNS well explains the observed sudden change in ee in the rigid gel.

In addition to the sudden ee change near the flowing-rigid gel border as a common feature shared by all the NSs employed, the ee variation profile reveals an interesting difference between the CD/CNN- and LM-based NSs. As can be seen from Fig. 3, the ee of 1E obtained upon sensitization with cyclic oligosaccharide-based 2-6 is not constant and appreciably varies across the sol to flowing gel region. This ee profile has been ascribed $^{36,37}$ to the gradual switching of the sensitization site

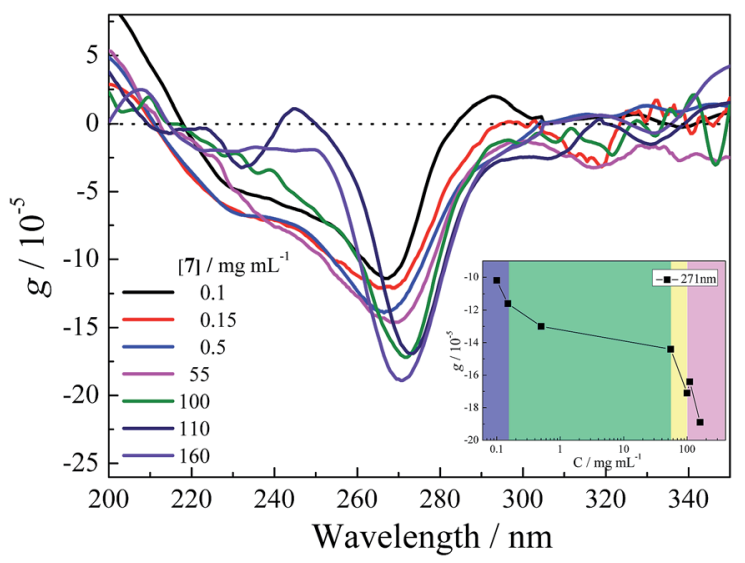

Fig. 4 Circular dichroism (anisotropy factor) spectra of LM-NS 7 in water at concentrations from 0.1 to $160 \mathrm{mg} \mathrm{mL}^{-1}$ measured in a quartz cell or by sandwiching the gel with two quartz plates. Inset: the $g$ factor at $271 \mathrm{~nm}$ as a function of concentration/phase of 7 . 
from inside to outside of the host cavity/concave in the crosslinked polymer at higher NS concentrations, where the cavity/ concave is progressively occupied by the pyromellitate moiety. In contrast, the ee profile for catenulate oligosaccharide-based 7 and $\mathbf{8}$ is practically flat in the same region, indicating that the chiral environment of the sensitization site is not greatly altered in LM-NS at least up to the flowing gel phase. We deduced that the hydrophobic binding/sensitizing site formed around the pyromellitate moiety is loose and flexile until the flowing gel phase, but starts to continuously change its shape and rigidity in the rigid gel phase. Without considering the continued shape/rigidity change, we cannot rationalize the sudden decrease and the subsequent recovery of ee observed upon sensitization with 7 (Fig. 3f).

As shown in Table 1 and Fig. 5a, the ee product obtained upon sensitization with 7 in 10\% methanolic water is also significantly phase-dependent, exhibiting a slow increase from $4.9 \%$ to $8.5 \%$ in the sol to early rigid gel region, but a subsequent sudden decrease down to $-5.4 \%$ in the late rigid phase. When the methanol content was increased up to $50 \%$, the ee

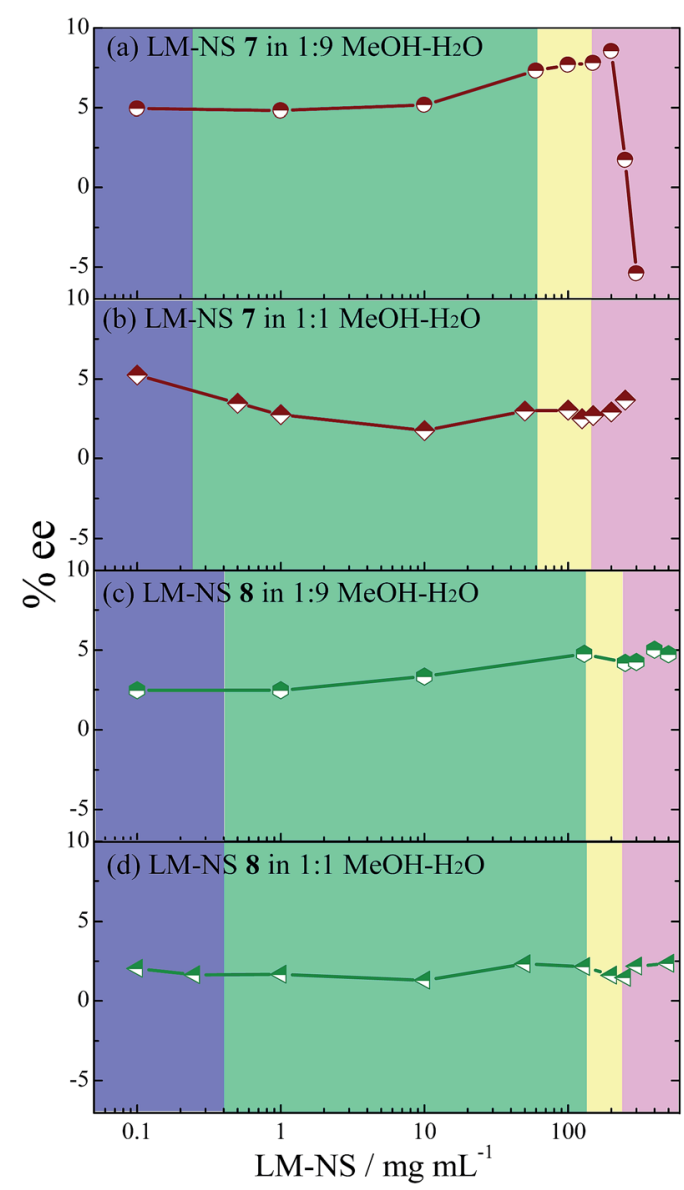

Fig. 5 ee profiles of $1 \mathrm{E}$ obtained upon enantiodifferentiating photoisomerization of $1 Z$ sensitized by LM-NSs 7 ( $a$ and $b$ ) and 8 ( $c$ and d) in aqueous solutions containing 10\% ( $a$ and $c$ ) and 50\% (b and d) methanol. The violet, green, yellow, and pink regions represent the sol, suspension, flowing gel, and rigid gel phases, respectively, where the phase transition concentrations in $1: 1 \mathrm{MeOH}-\mathrm{H}_{2} \mathrm{O}$ were essentially the same as in $1: 9 \mathrm{MeOH}-\mathrm{H}_{2} \mathrm{O}$. was lowered to some degree in the sol to flowing gel region and no significant ee change occurred in the rigid gel or any other phase (Fig. 5b). Similarly, appreciably lower ee and flat ee profiles were obtained for the photosensitization with 8 in 10\% or $50 \%$ methanolic water (Fig. $5 \mathrm{c}$ and d). These results reveal the critical roles of the hydrophobicity of the solvent and degree of cross-linking in this phase-sensitive photosensitization. The addition of methanol may loosen the hydrogel to make the hydrophobic sensitizing site more flexible, eventually leading to a global decrease in ee and phase-insensitive ee profile.

\section{Conclusions}

In this study, we prepared new linear maltodextrin-based nanosponges (LM-NSs) cross-linked by pyromellitate as photosensitizing media for the enantiodifferentiating photoisomerization of cyclooctene $(\mathbf{1 Z})$. By comparing the phaseevolution and photochirogenic behaviours of LM-NSs with those of cyclodextrin- and cyclic nigerosylnigerose-based CD/ CNN-NSs, we elucidated that the inherent host cavity/concave existing in CD/CNN-NSs does not affect the overall phaseevolution behaviour, but causes an appreciable drift in ee in the sol to flowing gel region through the inclusion of guest substrate 1Z. However, the chiral void space created around the mellitate linker in both LM-NSs and CD/CNN-NSs plays the major role in determining the stereochemical consequences of photoisomerization to $\mathbf{1 E}$, the ee of which shows a rather flat profile in the sol to flowing gel region but abrupt change(s) in the rigid gel phase, i.e. a sudden drop. These ee profiles disclose the nominal contribution of the host cavity/concave to the photochirogenesis in NS and also highlight the more crucial roles played by the phase-responsive chiral void space created in the cross-linked oligosaccharide polymers.

\section{Experimental}

\section{Materials}

Cyclooctene was purchased from Tokyo Chemical Industries and used after fractional distillation. LM-NSs 7 and 8 were prepared as follows. Typically, LM (4.89 g) of average molecular weight of ca. $12000 \mathrm{Da}$ was dissolved in DMSO (20 mL), to which trimethylamine $(5 \mathrm{~mL})$ and a proper amount of pyromellitic dianhydride (1.88 $\mathrm{g}$ for 7 or $3.76 \mathrm{~g}$ for 8 ) were added. Then, the mixture was stirred for $24 \mathrm{~h}$ until the solution gelated. The monolith block thus obtained was crushed in a mortar, washed with an excess amount of deionized water, and then filtered under vacuum. After air-drying, the whitish powder was collected and purified by Soxhlet extraction with acetone for $20 \mathrm{~h}$ to afford 7 in $91.7 \%$ yield and 8 in $98.6 \%$ yield. The other chemicals were commercially available and used as received.

\section{Instruments and methods}

The particle size distribution of LM-NS was determined by DLS in an aqueous solution at $25{ }^{\circ} \mathrm{C}$ by monitoring the scattered light at an angle of $173^{\circ}$ using a Zetasizer Nano instrument (Malvern Instrument, UK). The size and shape of the 
precipitates in the suspension were observed using an optical microscope (DN-107T digital microscope). UV-vis and circular dichroism spectra were obtained using JASCO V-560 and J-820 spectrometers, respectively. SEM images were obtained using a Hitachi tabletop microscope TM 3000.

\section{Photolysis}

In a typical run, $3 \mathrm{~mL}$ aqueous sample containing LM-NS and $\mathbf{1 Z}$ was placed in a quartz cell, purged with nitrogen gas, and then irradiated at $254 \mathrm{~nm}$ with two $11 \mathrm{~W}$ low-pressure mercury lamps (Philips TUV T5 4P-SE) in a Unisoku cryostat kept at a given temperature. No appreciable phase change or transition was observed upon irradiation. The photolyzed sample was poured into an aqueous $10 \% \mathrm{KOH}$ solution $(3 \mathrm{~mL})$, and the resulting mixture was extracted with pentane $(1 \mathrm{~mL})$. After the gas chromatographic (GC) analysis of an aliquot to determine the $E / Z$ ratio using a Shimadzu GC-2014 (CBP-20 column, $0.25 \mathrm{~mm} \phi \times$ $25 \mathrm{~m}, 60^{\circ} \mathrm{C}$ ), the organic layer was extracted with an aqueous silver nitrate solution $(2 \mathrm{~mL})$ at $0{ }^{\circ} \mathrm{C}$. The aqueous phase was washed twice with pentane $(1 \mathrm{~mL})$ and then added to an aqueous ammonia $(28 \%)$ solution at $0{ }^{\circ} \mathrm{C}$, and the liberated cyclooctene was extracted with pentane $(0.5 \mathrm{~mL})$. The ee was determined using a chiral GC equipped with a Supelco $\beta$-DEX 225 column $(0.25 \mathrm{~mm} \phi \times 30 \mathrm{~m})$.

\section{Acknowledgements}

This work was supported by the National Natural Science Foundation of China (No. 21402110, 21572142, 21372165, 21402129 and 21321061), Research Project Supported by Shanxi Scholarship Council of China (No. 2014-011), Scientific and Technological Innovation Programs of Higher Education Institutions in Shanxi (No. 2014108), State Key Laboratory of Polymer Materials Engineering, Grant No. sklpme 2014-2-06, Comprehensive Training Platform of Specialized Laboratory, College of Chemistry, Sichuan University and Japan Society for the Promotion of Science (No. 21245011 and 26620030).

\section{References}

1 Y. Inoue and V. Ramamurthy, Chiral Photochemistry, Dekker, New York, 2004.

2 C. Yang and Y. Inoue, Chem. Soc. Rev., 2014, 43, 4123.

3 V. Ramamurthy and J. Sivaguru, Chem. Rev., 2016, 116, 9914. 4 M. Oelgemöller and N. Hoffmann, Org. Biomol. Chem., 2016, 14, 7392.

5 A. G. Griesbeck and U. J. Meierhenrich, Angew. Chem., Int. Ed., 2002, 41, 3147.

6 C. Müller and T. Bach, Aust. J. Chem., 2008, 61, 557.

7 N. J. Turro, V. Ramamurthy and J. C. Scaiano, Modern Molecular Photochemistry of Organic Molecules, Wiley Online Library, 2012.

8 G. S. Hammond and R. S. Cole, J. Am. Chem. Soc., 1965, 87, 3256.

9 Y. Inoue, T. Yokoyama, N. Yamasaki and A. Tai, Nature, 1989, 341, 225.
10 Y. Inoue, H. Ikeda, M. Kaneda, T. Sumimura, S. R. L. Everitt and T. Wada, J. Am. Chem. Soc., 2000, 122, 406.

11 A. Bauer, F. Westkaemper, S. Grimme and T. Bach, Nature, 2005, 436, 1139.

12 R. Brimioulle and T. Bach, Science, 2013, 342, 840.

13 J. Sivaguru, A. Natarajan, L. S. Kaanumalle, J. Shailaja, S. Uppili, A. Joy and V. Ramamurthy, Acc. Chem. Res., 2003, 36, 509.

14 H. Saito, T. Mori, T. Wada and Y. Inoue, J. Am. Chem. Soc., 2004, 126, 1900.

15 J. Gui, Z. Yan, Y. Peng, J. Yi, D. Zhou, D. Su, Z. Zhong, G. Gao, W. Wu and C. Yang, Chin. Chem. Lett., 2016, 27, 1017.

16 C. Yang, Chin. Chem. Lett., 2013, 24, 437.

17 Y. Inoue, S. F. Dong, K. Yamamoto, L.-H. Tong, H. Tsuneishi, T. Hakushi and A. Tai, J. Am. Chem. Soc., 1995, 113, 2793.

18 C. Yang, T. Mori, Y. Origane, Y. H. Ko, N. Selvapalam, K. Kim and Y. Inoue, J. Am. Chem. Soc., 2008, 130, 8574.

19 C. Yang, C. Ke, W. Liang, G. Fukuhara, T. Mori, Y. Liu and Y. Inoue, J. Am. Chem. Soc., 2011, 133, 13786.

20 J. Yao, Z. Yan, W. Wu, C. Yang, M. Nishijima, G. Fukuhara, T. Mori and Y. Inoue, J. Am. Chem. Soc., 2014, 136, 6916.

21 Q. Wang, C. Yang, C. Ke, G. Fukuhara, T. Mori, Y. Liu and Y. Inoue, Chem. Commun., 2011, 47, 6849.

22 G.-H. Liao, L. Luo, H.-X. Xu, X.-L. Wu, L. Lei, C.-H. Tung and L.-Z. Wu, J. Org. Chem., 2008, 73, 7345.

23 L. Luo, S.-F. Cheng, B. Chen, C.-H. Tung and L.-Z. Wu, Langmuir, 2010, 26, 782.

24 A. Joy, S. Uppili, M. R. Netherton, J. R. Scheffer and V. Ramamurthy, J. Am. Chem. Soc., 2000, 122, 728.

25 J. Sivaguru, T. Shichi and V. Ramamurthy, Org. Lett., 2002, 4, 4221.

26 J. Sivaguru, S. Jockusch, N. J. Turro and V. Ramamurthy, Photochem. Photobiol. Sci., 2003, 2, 1101.

27 S. Asaoka, T. Wada and Y. Inoue, J. Am. Chem. Soc., 2003, 125, 3008.

28 M. Nishijima, T. Wada, T. Mori, T. C. S. Pace, C. Bohne and Y. Inoue, J. Am. Chem. Soc., 2007, 129, 3478.

29 J. Mizoguchi, Y. Kawanami, T. Wada, K. Kodama, K. Anzai, T. Yanagi and Y. Inoue, Org. Lett., 2006, 8, 6051.

30 T. Bach, H. Bergmann, B. Grosch and K. Harms, J. Am. Chem. Soc., 2002, 124, 7982.

31 C. Müller, A. Bauer, M. M. Maturi, M. C. Cuquerella, M. A. Miranda and T. Bach, J. Am. Chem. Soc., 2011, 133, 16689.

32 Z. Yan, Q. Huang, W. Liang, X. Yu, D. Zhou, W. Wu, J. Chruma and C. Yang, Org. Lett., 2017, 19(4), 898-901.

33 A. Dawn, T. Shiraki, S. Haraguchi, H. Sato, K. Sada and S. Shinkai, Chem.-Eur. J., 2010, 16, 3676.

34 M. Shirakawa, N. Fujita, T. Tani, K. Kaneko and S. Shinkai, Chem. Commun., 2005, 4149.

35 Y. Ishida, Y. Kai, S.-y. Kato, A. Misawa, S. Amano, Y. Matsuoka and K. Saigo, Angew. Chem., Int. Ed., 2008, 47, 8241.

36 W. Liang, C. Yang, D. Zhou, H. Haneoka, M. Nishijima, G. Fukuhara, T. Mori, F. Castiglione, A. Mele, F. Caldera, F. Trotta and Y. Inoue, Chem. Commun., 2013, 49, 3510. 
37 X. We, W. Liang, W. Wu, C. Yang, F. Trotta, F. Caldera, A. Mele, T. Nishimoto and Y. Inoue, Org. Biomol. Chem., 2015, 13, 2905.

38 W. Liang, C. Yang, M. Nishijima, G. Fukuhara, T. Mori, A. Mele, F. Castiglione, F. Caldera, F. Trotta and Y. Inoue, Beilstein J. Org. Chem., 2012, 8, 1305.

39 R. Cavalli, F. Trotta and W. Tumiatti, J. Inclusion Phenom. Macrocyclic Chem., 2006, 56, 209.

40 F. Trotta, M. Zanetti and R. Cavalli, Beilstein J. Org. Chem., 2012, 8, 2091.
41 S. Swaminathan, R. Cavalli, F. Trotta, WIREs Nanomedicine and Nanobiotechnology, 2016, vol. 8, pp. 579-601.

42 C. Yang, T. Mori, T. Wada and Y. Inoue, New J. Chem., 2007, 31, 697.

43 R. Lu, C. Yang, Y. Cao, Z. Wang, T. Wada, W. Jiao, T. Mori and Y. Inoue, J. Org. Chem., 2008, 7695.

44 R. Lu, C. Yang, Y. Cao, Z. Wang, T. Wada, W. Jiao, T. Mori and Y. Inoue, Chem. Commun., 2008, 374.

45 G. Fukuhara, T. Mori, T. Wada and Y. Inoue, Chem. Commun., 2005, 4199. 